\title{
Prácticas de los estudiantes en el espacio de la cafetería de un colegio público
}

\author{
Student practices in the space of a public school cafeteria
}

\section{Práticas dos alunos no espaço da cantina de um colégio publico}

\author{
Jhon Alexánder Monsalve Flórez \\ Magister en Semiótica \\ Universidad Industrial de Santander \\ monsalve-jhon@hotmail.com
}

\section{Resumen}

Este artículo de reflexión surge de una investigación cualitativa, orientada desde el modelo hermenéutico-interpretativo, que utiliza la técnica de observación directa de las Ciencias Sociales. La pesquisa se enmarca dentro de la línea "Prácticas pedagógicas" del Grupo de Investigación Cultura y Narración en Colombia (Cuynaco), adscrito a la Escuela de Idiomas de la Universidad Industrial de Santander; así mismo, recibe el apoyo metodológico de la Maestría en Métodos y Técnicas de Investigación Social, de la Escuela de Historia de la misma universidad. El objetivo de la pesquisa es analizar las prácticas de los estudiantes cuando utilizan el espacio de la cafetería en un colegio público de Girón, Santander. Para ello, se propone, luego de la observación, el proceso de categorización de la información recolectada, mediante el análisis de contenido. Los hallazgos se describen en función de cuatro categorías: a) Comportamientos habituales de los sujetos en las filas para compra de productos, b) Comportamientos habituales de los sujetos que no compran en cafetería, pero utilizan el espacio, c) Relaciones de poder y d) Inestabilidad de la concurrencia estudiantil en la cafetería. Se concluye, entre otras cosas, que la práctica más común de los estudiantes en el espacio de la cafetería es el juego, aunque, por su naturaleza, este sea valorado negativamente por los docentes y directivos de la institución.

Palabras clave: Observación, colegio, espacio, juego. 


\begin{abstract}
This reflection article arises from a qualitative investigation, oriented from the hermeneutic-interpretive model, which uses the direct observation technique of the Social Sciences. The research is framed within the line "Pedagogical practices" of the Research Group Culture and Narration in Colombia (Cuynaco), attached to the School of Languages of the Industrial University of Santander; Likewise, it receives the methodological support of the Master in Methods and Techniques of Social Research, from the School of History of the same university. The objective of the research is to analyze the students' practices when they use the cafeteria space in a public school in Girón, Santander. To do this, it is proposed, after observation, the categorization process of the information collected, through content analysis. The findings are described in terms of four categories: a) Habitual behaviors of the subjects in the lines to buy products, b) Habitual behaviors of the subjects who do not buy in the cafeteria, but use space, c) Power relations and d) Instability of student attendance in the cafeteria. It is concluded, among other things, that the most common practice of students in the cafeteria space is play, although, by its nature, it is negatively valued by the teachers and managers of the institution.
\end{abstract}

Keywords: Observation, school, space, game.

\title{
Resumo
}

Esse artigo de reflexão emerge de uma pesquisa qualitativa a partir do modelo hermenêutico interpretativo que usa a técnica de observação direta das Ciências Sociais. A pesquisa se enquadra na linha "Práticas Pedagógicas" do Grupo de Pesquisa Cultura e Narração em Colômbia (Cuynaco) vinculado à Escola de Línguas da Universidad Industrial de Santader, embora recebe apoio metodológico do Mestrado em Métodos e Técnicas de Investigação Social da Escola de História da mesma Universidade. O objetivo da pesquisa é analisar as práticas dos alunos quando usam o espaço da cantina num Colégio público do Município de Girón, Santander na Colômbia. Assim propõe-se logo da observação, um processo de categorização da informação recolhida, através do analise de conteúdo. Os achados se descrevem em função de quatro categorias: a) comportamentos habituais dos sujeitos nas filas para compras de produtos, b) Comportamentos habituais dos sujeitos que não compram na cantina, mas usam o espaço, c) Relações de poder e d) Inconstância na assistência dos alunos à cantina. Conclui-se, entre outras coisas, que a prática mais comum dos alunos no espaço da cantina é brincar, ainda que por sua natureza tenha-se avaliado dito comportamento como negativo pelos professores e diretivos da Instituição.

Palavras-chave: Observação, colégio, espaço, brincar.

\section{Introducción}

En el ámbito de la investigación cualitativa, la observación directa y sistematizada se configura como una técnica que aporta información importante para la comprensión de problemas sociales. Desde Postic y De Ketele (2000), la observación es comprendida como una "operación de selección y de estructuración de datos de modo que quede patente una red de significaciones" (p. 22), es decir, la acción de observar trasciende los límites de la mirada superficial no analítica: permite, por el contrario, reconocer datos que lleven a entender la red de sentido de los grupos sociales observados. La definición que, por su parte, proponen Campos y Lule (2012) se emparenta con la anterior: “(...) definiremos a la observación como una técnica que mediante la aplicación de ciertos recursos permite la organización, coherencia y economía 
de los esfuerzos realizados durante el desarrollo de una investigación" (p. 49). En el mismo sentido, Herrero (1997), luego de una descripción teórica del concepto, concluye que las propuestas halladas en su investigación tienden a considerar "el instrumento básico de la misma [la observación] a la capacidad de percepción e interpretación del ser humano" (p. 2). En esencia, la observación es un proceso sistemático y organizado que busca la comprensión de los comportamientos humanos en contextos particulares, a partir de objetivos estipulados que surgen de algún problema social; en el caso del estudio que se presenta en el presente artículo, la observación es una técnica de investigación cualitativa que facilita el estudio de las conductas, como parte de las actitudes de los estudiantes (Rosenberg y Hovland, 1960; Pacheco, 2002), en la cafetería de un centro educativo de Girón, Santander.

En esta institución los docentes y directivos ven con preocupación el comportamiento inadecuado de los estudiantes durante los momentos recreacionales, que, desde una dimensión social y pedagógica, se comprenderían como el tiempo complementario de formación de los educandos con respecto a sus relaciones humanas (De Castro, 2018). Para garantizar una convivencia sana a la hora del descanso, los docentes perciben, por turnos semanales, las actitudes negativas del estudiantado con el fin de orientar su comportamiento. $\mathrm{Al}$ respecto, el trabajo realizado por Artavia (2014) expone las consecuencias positivas del proceso de supervisión del profesorado no solo en el aula de clase, sino también en los demás espacios físicos de la institución. En concordancia con esta investigadora, pareciera que las acciones de vigilancia escolar son necesarias para fomentar una cultura de la formación académica integral, en donde el comportamiento y el trato mutuo estudiantil se orientenhacia acciones pacíficas.

Compartiendo una razón similar, surgen estudios que propenden —más allá del acto punitivo o de orientación profesoral dialogantepor comprender las razones por las cuales los estudiantes se comportan de manera inapropiada en el momento recreacional. Chaves (2013) plantea que el juego es fundamental para los estudiantes durante el descanso, pero se cuestiona, a su vez, por las implicaciones de estos actos recreativos $\mathrm{y}$, por tanto, propone alternativas de juegos para el estudiantado. López (2017) considera que los actos de violencia en el recreo deben comprenderse como posibilidades para reorientar el ser de los niños y jóvenes, que se comportan de manera inapropiada a razón del contexto violento en el que son educados.

Otras investigaciones se relacionan de manera directa con el uso de la técnica de observación en la hora del recreo escolar. Jarauta (2017) observa las actividades y conflictos de los niños del ciclo infantil en una institución española y concluye que los estudiantes, si bien experimentan trances con sus compañeros, se rescata la creatividad en la propuesta de juegos en el espacio recreacional. Por su parte, Peñaranda (2017) halla un uso interesante en dos zonas recreacionales de la institución educativa que observa: en una de ellas, socializa pasivamente cierto grupo de estudiantes, mientras que en la otra zona los comportamientos suelen ser violentos. Buritica (2014) sitúa en manos de los docentes la responsabilidad de disminuir los niveles de violencia en la hora del recreo e invita a entender el tiempo recreacional como una oportunidad para la orientación ética del estudiantado en pro de una mejor sociedad.

Lo anterior constata un problema de violencia que se presenta en el momento recreacional de los estudiantes. Ningún trabajo ha contemplado, hasta la fecha, la posibilidad de analizar las prácticas estudiantiles en ciertos espacios específicos del recreo, tales como la cafetería, lugar en el que también se socializa y se presenta violencia, según lo que se observa en la institución estudiada en esta pesquisa. Por cuestiones de delimitación, y con el fin de aportar en la comprensión del uso de espacios específicos durante el tiempo del recreo, se propone estudiar 
el comportamiento de los sujetos escolares, específicamente, en lo relacionado con el uso de la cafetería en un colegio de Girón, Santander. Metodológicamente, se procede, en primera instancia, a realizar observaciones hasta que se sature la información de los hechos objeto de análisis; paso seguido, se plantean categorías tendientes a la comprensión de las actitudes comportamentales de los estudiantes y de los profesores de vigilancia dentro de los límites de la cafetería institucional. Por último, se concluye que la cafetería es un espacio más de juego y de relaciones comunicativas afectivas para los estudiantes.

\section{Descripción de la población observada y del lugar}

La población estudiada comprende, en una primera etapa, cerca de 500 estudiantes y, en el segundo momento de la pesquisa, 1004 estudiantes de la jornada única de un colegio público del municipio de Girón, Santander. Los educandos pertenecen a los estratos socioeconómicos 1, 2 o 3 y cursan alguno de los grados académicos de primero hasta décimo. En las primeras cuatro observaciones, los estudiantes con mayor vulnerabilidad social recibían, durante el descanso y por parte del Programa de Alimentación Escolar (PAE), un complemento alimenticio en horas de la tarde. Desde la quinta observación, cuando hubo cambio de planta en el colegio, todos los estudiantes fueron beneficiados con el almuerzo escolar por el mismo programa estatal.

En principio, es necesario realizar dos descripciones del espacio de la cafetería del Colegio Villas de San Juan, debido a que este lugar, con el cambio de planta, pasa a ser reubicado a la vera de los baños del primer piso y al lado del restaurante escolar, luego de estar al lado de la portería antigua y aledaño a las escaleras de una de las torres de salones.

La cafetería de la planta antigua se ubica, en su momento, en la entrada después de la portería. Se encuentra precisamente en medio de la nueva planta y de la antigua. Mide tres metros de ancho y dos de fondo. Es azul. Está ubicada específicamente entre las escaleras de la planta antigua y los pasillos de la planta nueva. El espacio que hay entre la cafetería y la pared de la planta antigua no supera los cuatro metros. Es, justamente, el espacio en que los estudiantes pasean, compran y comparten. Con facilidad, desde la zona superior de la nueva planta, los estudiantes pueden ver el techo del lugar y la aglomeración de sus compañeros mientras compran los respectivos productos.

La cafetería de la nueva planta es exactamente la misma en estructura, tamaño y color. Los encargados de ella optan por trasladarla tal cual al lado del restaurante escolar antiguo y delante de los sanitarios del primer piso. En la parte izquierda del lugar, se halla la cancha de fútbol y básquet, que, como se evidencia más abajo, no interfiere en los usos que hacen del espacio los estudiantes y profesores. La ventaja que, sin duda, posee esta reubicación radica en el espacio para la fila y la erradicación del hacinamiento.

\section{Metodología}

La técnica observacional, tal como se concibe en este trabajo, subyace a un enfoque cualitativo de la investigación, ligado, a su vez, al paradigma hermenéutico- interpretativo de la ciencia. Siguiendo a Rodríguez, Gil y García (1998), se tienen en cuenta cuatro fases para el desarrollo de la pesquisa: en primer lugar, la fase preparatoria, en la cual, luego de reconocer el problema de investigación, se plantean las actividades correspondientes para su respectiva comprensión. En esta fase, se proponen los formatos de observación y se programan las actividades correspondientes en un cronograma tentativo.

El formato de observación demanda datos fundamentales para el desarrollo de la pesquisa: fecha, lugar, hora y participantes; actores y acciones observados; descripción detallada de las prácticas de los estudiantes durante el uso del 
espacio de cafetería; comentarios analíticos y apreciaciones finales.

Así mismo, se selecciona el espacio en donde se hará el proceso de observación. En segundo lugar, la fase de trabajo de campo consiste en llevar a cabo lo planificado en la fase anterior. El proceso se realiza hasta que haya saturación de datos y tras un objetivo constante: observar las prácticas sociales de los estudiantes en la cafetería de un colegio público de Girón, Santander, a la hora del descanso. Estas actividades se desarrollan en dos espacios diferentes, debido a que, durante el tiempo en que se llevan a cabo las observaciones, es entregada la nueva planta de la institución. De once observaciones, cuatro son desarrolladas en la planta antigua y siete, en la planta nueva. En la medida en que se observan las prácticas de uso del espacio de cafetería, el investigador toma apuntes de audio que se transcriben después. Es importante aclarar que, desde Gold (1958), el investigador es un observador-como-participante, es decir, conocido por los estudiantes, pero distanciado de la población observada.

La fase analítica surge de la categorización de la información, a partir del estudio de contenido, en el cual lo observado o estudiado es "sometido a un análisis, a una fragmentación de componentes con sentido completo e independiente llamados unidades de información, o unidades de análisis, las cuales a su vez corresponden a categorías específicas" (Valbuena, 2013, p. 214). Para este caso, se refiere, entonces, al análisis del contenido que surge de las observaciones y que es organizado e interpretado, tal como sugiere la teoría al respecto (Herrero, 1997; Postic y De Ketele, 2000; Campos y Lule, 2012). La última etapa es informativa: los hallazgos de la investigación se presentan ante la comunidad directamente interesada y ante la ciencia, con el fin de reflexionar y, llegado el caso, actuar.

\section{Particularidades metodológicas de observación}

En la planta antigua, el descanso de primaria inicia a las 2:00 p.m. y culmina a las 2:26 p.m., mientras que el de bachillerato inicia a las 2:56 p.m. y termina a las 3:16 p.m. En la nueva planta, el recreo empieza a las 9:00 a.m. para primaria y a las 10:00 a.m. para secundaria y dura, exactamente, 30 minutos. En las primeras cuatro observaciones, por disponibilidad de tiempo, no se toma registro de los usos que hacen de la cafetería los niños de primaria; solo se tienen en cuenta las prácticas de los estudiantes de bachillerato. Sin embargo, en la nueva planta, con mayor disponibilidad de tiempo, el investigador toma apuntes de los comportamientos de los niños de primaria a la hora del recreo y, así mismo, continúa estudiando las prácticas de los estudiantes de bachillerato en el nuevo espacio de cafetería. Metodológicamente, esto plantea un reto importante en la categorización del contenido: por una parte, se contrastan las prácticas de los mismos estudiantes en dos espacios diferentes de la cafetería (planta nueva y planta antigua) y, por otra, se comparan los comportamientos de estudiantes de primaria y de bachillerato en el nuevo espacio de cafetería.

Por último, con respecto a las consideraciones éticas del estudio, es importante aclarar que, según la Resolución 8430 de 1993, es una investigación sin riesgo para los sujetos observados. En vista de que se analizan las prácticas en el uso de la cafetería y que tal acción no afecta, de ninguna manera, el bienestar ni la salud de los estudiantes, los directivos del colegio otorgan el permiso para que el docente investigador realice sus procesos de recolección de información.

\section{Hallazgos}

Luego de 11 observaciones (ya se ha aclarado que fueron solo 11 por motivos de saturación de información) de las prácticas de estudiantes y profesores en la cafetería de un colegio público de Girón, surgen hallazgos importantes sobre formas de comportamiento de los sujetos sociales que hacen uso del espacio de la cafetería. La descripción que se presenta en los siguientes párrafos hace alusión a cuatro categorías que 
pueden resumir tales actitudes de estudiantes y profesores: 1) Comportamientos habituales de los sujetos en las filas para compra de productos, 2) Comportamientos habituales de los sujetos que no compran en cafetería, pero utilizan el espacio, 3) Relaciones de poder, 4) Inestabilidad de la concurrencia estudiantil en la cafetería.

\section{Comportamientos habituales de los sujetos en las filas para compra de productos}

En primer lugar, se evidencia que los estudiantes actúan de manera agresiva, pero jocosa, durante la compra de los productos en cafetería. Hay recurrencias de empujones, golpes en la cabeza con las manos, gritos agresivos e insultos, propios de la dinámica de hacer la fila en esta institución y seguidos de risas y saludos amigables. Cabe aclarar que estos comportamientos son más habituales en los estudiantes de sexto grado, aunque se observa que, en algunas ocasiones, los de niveles superiores caen en prácticas similares: "Los estudiantes de sexto grado continúan los juegos violentos. A pesar del espacio, que ahora es más amplio, siguen las prácticas de agresión de los estudiantes, que, para ellos, hacen parte de los juegos propios de la hora del recreo" (Nota 3 de la Observación de campo 7).

Lo anterior permite la creación de una categoría teórica emergente que, durante el proceso de la investigación y hasta la fecha, no se ha podido encasillar dentro de algún concepto semiótico (Fontanille, 2001) o dentro de alguna temática de la prosaica (Mandoki, 2006). Por tal motivo, en el marco de la investigación aquí expuesta, se propone, de manera tentativa, el concepto "Disfemismo somático", para hacer alusión a las actitudes de violencia entre estudiantes, las cuales son productos de afectos demostrados en público. El término teórico deviene del concepto lingüístico disfemismo y de las relaciones somáticas entre sujetos. En primera instancia, el disfemismo es antónimo de eufemismo y, por tanto, en lugar de que ciertas palabras o expresiones sean consideradas como tabúes, el hablante intensifica tales enunciados con fines pasionales positivos. En términos de Canoy, citado por Carreter (1998):

El disfemismo es un concepto para liberarse de la actitud admirativa o respetuosa que gravita, en general, sobra la humanidad media. Consiste, sobre todo, en la sustitución de los términos nobles, o simplemente normales, por expresiones tomadas en dominios más vulgares, más familiares, más regocijantes (p. 147).

Con lo anterior, llama la atención el intercambio de lexemas o expresiones nobles por enunciados vulgares, pero con cierta relación familiar y con fines regocijantes. En pocas palabras, un sujeto podría referirse a un allegado con palabras vulgares, pero que, pragmáticamente, sean comprendidas como afectivas. Lo que se pretende, desde esta perspectiva del término, es equiparar tales acciones lingüísticas de fines pasionales positivos con las actitudes somáticas de los estudiantes que hacen la fila y golpean a sus compañeros. Tales relaciones sociales con el cuerpo son comprendidas por Greimas y Courtés (1990) como un hacer somático pragmático, en el que se "alude a una actividad corporal programada" (p. 394), es decir, a un hacer del sujeto que trasciende los fines comunicativos de los gestos, para dar sentido, en este caso, a los "golpes afectivos" de los estudiantes hacia sus compañeros en horario recreacional. En últimas, los sujetos observados dejan un comportamiento "noble" o "normal" por actuar de manera violenta, pero amigable, con los sujetos con los cuales se relacionan.

Por otra parte, se evidencia cierto aprovechamiento de algunos estudiantes que llegan tarde a la fila con el objetivo de comprar más rápidamente que aquellos que llegan temprano; por esta razón, se crean filas improvisadas que, aun así, son tenidas en cuenta por los señores que atienden este espacio escolar: "Desde esta perspectiva, se alcanzan a percibir 
hasta seis filas. Dos de ellas son las ideales; las demás filas son amorfas, y, sin embargo, los que atienden hacen caso omiso de esta desorganización" (Nota 4 de la Observación de campo 3).

Dentro de esta misma categoría, es importante resaltar un cambio positivo que hubo luego de que la institución ampliara su espacio con una planta mayor que favorecería, entre otras cosas, el horario de única jornada en el colegio. La cafetería cambió de lugar y con ella el orden de las filas tendió a mejorar, aunque no deja de verse una que otra fila improvisada. Se nota un mejor orden debido a que el espacio es mucho mayor que el utilizado en la planta anterior: la fila de los hombres suele ser la más extensa y la de las mujeres tiende a ser la más corta, pero también la más ordenada. Se acepta, con este cambio, una tercera fila mixta para compra exclusiva de productos helados, la cual no existía, por cuestiones de espacio, en la planta anterior: "Hay dos lugares de la cafetería por los que ahora atienden a los estudiantes [justo en frente, donde se forman las filas principales, y en la parte izquierda, en la venta de helados]. La persona nueva de la cafetería (ahora atienden tres) está encargada de manera exclusiva de la nueva puerta de atención y venta" (Nota 3 de la Observación de campo 6).

De la misma manera, hay diferencias importantes en cuanto al comportamiento de los estudiantes de bachillerato comparado con las actitudes de los estudiantes de primaria. Estos últimos suelen ser más ordenados y están todo el tiempo acompañados por sus profesores: mientras que en bachillerato solo son cuatro los profesores de vigilancia, en primaria cada docente es responsable de su grupo, tal como se evidencia en la siguiente nota:

Los docentes de primaria están bastante atentos al comportamiento de los estudiantes a la hora del descanso. Aunque no hacen uso de la cafetería para comprar sus productos, vigilan las actitudes negativas de los estudiantes y esto conlleva por parte de los niños un buen comportamiento (Nota de análisis 2 de la Observación de campo 6).

Por último, dentro de esta misma categoría, es importante destacar el uso que hacen los docentes de la cafetería: hay muy poca participación de estos sujetos sociales tanto para compra de productos como para transitar. En la planta antigua, se veía, de vez en cuando, a los docentes pasar por la cafetería, aunque no fueran con fines de compra; en la nueva planta, los docentes observan desde los pisos superiores y, en panorámica, pueden reconocer momentos de conflicto dentro o fuera del espacio de la cafetería. Por lo general, los maestros traen sus alimentos o los compran fuera de la institución en horas del descanso.

\section{Comportamientos habituales de los sujetos que no compran en cafetería, pero utilizan el espacio}

En la planta antigua, por motivos de hacinamiento, los estudiantes que no compraban productos se paseaban por la cafetería conversando o jugando a correr y golpearse.

En esas observaciones, se evidencia que la cafetería no es un espacio exclusivo para comprar productos, sino también para hablar y jugar o, como sucedía en varias ocasiones, transitar como puente para salones superiores o portería del colegio:

Durante el descanso, la cafetería permanece concurrida. Entre 25 y 40 estudiantes, hacen fila o se pasean por tal lugar buscando esparcimiento. Los que no hacen fila juegan a empujarse, ríen a carcajadas, se burlan, en algún momento, de un estudiante que resbaló en la escalera (Nota 6 de la Observación de campo 1).

En la nueva planta, la cafetería se usa principalmente para comprar productos. Los espacios ahora son más amplios y esto lleva a que los estudiantes utilicen la cafetería de manera exclusiva para su fin primario: "El espacio de la cancha hace que la aglomeración no sea tan 
significativa en la cafetería" (Nota 6 de la Observación de campo 6).

No todos los estudiantes de bachillerato compran productos en este lugar: muchos de ellos no tienen dinero o traen comida de la casa (aunque es poco común ver esto en secundaria); mientras los demás compran, estos estudiantes suelen pasearse por la nueva planta haciendo uso de la cancha, muy cercana a la cafetería, o jugando trompo a escasos veinte metros de las filas: "A la hora del descanso, los estudiantes se dirigen a la zona de la cancha. La mayoría no compra los productos de este lugar" (Nota 1 de la Observación de campo 7). Los niños de primaria que no hacen uso de la cafetería se sientan con sus loncheras cerca de la cafetería a la espera de algún compañero para hablar: "Luego de comprar, los estudiantes se dispersan por la institución; no ocurre lo mismo con los compradores en grados de primaria, quienes se sientan en los andenes cercanos a la cafetería y que colindan con los baños del primer piso" (Nota 5 de la Observación de campo 7).

\section{Relaciones de poder}

Ante todo, se parte del hecho de que cualquier relación social determina formas de poder. Así como se hace explícita, desde la jerarquía administrativa o académica, la relación de poder entre los actores de una institución, los estudiantes, en su rol de seres sociales, intercambian sentidos desde niveles diferentes. $\mathrm{Al}$ respecto, Burbules (1989) concibe, luego de asociar este tema con la estructura educativa, que "las relaciones de poder son inherentes a nuestra sociedad: entre clases, entre sexos y entre varios grupos raciales, étnicos y religiosos" (p. 25).

En el espacio de cafetería, se evidencian relaciones de poder de tres tipos. En primer lugar, los estudiantes que golpean a los demás se configuran como sujetos de poder, capaces de realizar actos de subestimación hacia compañeros que realizan la fila de la cafetería en puestos más cercanos a la plataforma física de atención al cliente. Aunque se ha reiterado que estas acciones tienen fines lúdicos, aparentemente consensuados por los estudiantes que participan del juego, no por ello el acto de poder se difumina.

Estas formas de poder se evidencian en las siguientes notas de campo: "Los estudiantes se empujan unos a otros. Se ríen. Gritan para hacer respetar su espacio. Además, como práctica particular, sobre todo en niños de sexto grado, surgen golpes en la cabeza, conocidos por la comunidad como "calvazos" (Nota 4 de la Observación de campo 1) y "Hoy es más notorio el hecho de que se apretujen los estudiantes. Hay estudiantes pegados contra las latas de la caseta riéndose porque los de atrás empujan" (Nota 6 de la Observación de campo 4).

En segundo lugar, la relación de poder "virtualizada" y comandada por los profesores durante la denominada "vigilancia de estudiantes", entendida como el acto obligatorio de los docentes de estar atentos a cualquier circunstancia de indisciplina o accidente en el desarrollo del descanso estudiantil. Los profesores encargados en los días de las observaciones en la planta antigua se veían pasar de manera esporádica, pero no tenían intenciones de llamar la atención a los estudiantes por su comportamiento durante la realización de la fila en cafetería. En lo ideal, existe una relación de poder entre docentes y estudiantes en la hora de descanso, pero queda virtualizada debido a que, según parece, el acto de vigilar se traslada a otros espacios institucionales. En el comentario de la segunda observación de campo quedan en evidencia estos aspectos y, de paso, se conecta con el último tipo de relación de poder:

Los profesores, incluso los de vigilancia, no transitan por el espacio de la cafetería. Se presentan empujones y juegos bruscos que acarrean potenciales problemas de convivencia. El espacio de la cafetería es un espacio relativamente vacío de profesores. La responsabilidad de lo que pase en las filas parece recaer en los que atienden la cafetería (Comentario único de la Observación de campo 2). 
En última instancia, la relación de poder más evidente ocurre en el intercambio económico: los que atienden se muestran como sujetos de poder, por una parte, en relación con su rol de propietarios del lugar y de los productos que necesitan los estudiantes y, por otra, en relación con la autoridad que se hace necesaria para la organización de las compras respectivas.

Los que atienden la cafetería no prestan atención a las agresiones físicas de manera particular, pero sí orientan discursos de orden de manera general, sobre todo cuando se pone en riesgo un bien de su propiedad:

En tres momentos, los señores que atienden la cafetería llaman la atención de los estudiantes porque, al parecer, el desorden de las filas produce una amenaza con respecto al recipiente de las empanadas.Constantemente, los encargados de la cafetería piden orden a los estudiantes (Nota 5 de la Observación de campo 1).

Otra manera en que se evidencia el poder de los que encargados de la cafetería consiste en la decisión de atender las filas improvisadas o negarse a ello. Una de las insistencias más recurrentes de los estudiantes en la planta antigua radicaba en que los atendieran por la parte izquierda, en donde eran ubicados los productos helados.

Ante esta situación, "cabe aclarar que los que atienden se niegan a prestar atención a estos estudiantes" (Nota 3 de Observación de campo $3)$.

Curiosamente y en el mismo sentido, los que atienden no hacen lo mismo con las filas amorfas improvisadas: "Desde esta perspectiva, se alcanzan a percibir hasta seis filas. Dos de ellas son las ideales; las demás filas son amorfas, y, sin embargo, los que atienden hacen caso omiso de esta desorganización" (Nota 4 de la Observación de campo 3).

\section{Inestabilidad de la concurrencia estudiantil en la cafetería}

Resulta complejo determinar las causas de por qué la aglomeración en cafetería disminuía o aumentaba en ciertos días. Existen algunas razones sustentables con hechos; por ejemplo, en la cuarta observación de campo, se evidencia una cantidad importante de estudiantes haciendo fila, que trasciende los límites normales de saturación. No obstante, esto se explica por la ausencia de uno de los encargados de atender la cafetería: "El hecho de que hubiera faltado el señor que atiende y la responsabilidad hubiera recaído en una sola persona hace que la saturación del lugar sea desbordante" (Punto 1 del análisis en la Observación de campo 4).

Normalmente, se presenta cierta saturación en los primeros minutos de iniciado el descanso, luego, una ausencia de estudiantes y, por último, al finalizar la hora recreacional, otra saturación. Por estas recurrencias, surge la primera nota del cuarto diario de campo: "A las 2:52 p.m., hay solo cinco estudiantes comprando en la cafetería. Este hecho es poco común. Normalmente, a esta hora la cafetería está copada" (Nota 1 de la Observación de campo 4). En la nueva planta, la concurrencia es alta en los primeros días, pero, luego, todo apunta que, a raíz de ciertos beneficios alimenticios que reciben los estudiantes, la cantidad de compradores disminuye. El día de la inauguración de la nueva planta se evidencia lo siguiente: "La nueva planta del colegio entra a funcionar. Aunque es la misma cantidad de estudiantes que sale a descanso a las 10:00 a.m., en esta ocasión se evidencia una aglomeración importante en la cafetería" (Nota 1 de la Observación 5). Días después se constata que los estudiantes disminuyen el nivel de compra en la cafetería.

Justamente, en el octavo diario de campo se describe la poca afluencia de estudiantes en la cafetería, pero, en esa ocasión, se comprende porque dos cursos de sexto grado no asisten al plantel educativo. Ante este hecho, se propone como hipótesis que la poca concurrencia es 
resultado de la ausencia de estudiantes que suelen comprar productos en la cafetería. No obstante, este lugar permanece sin mucha conglomeración durante el descanso del día siguiente, a pesar de la asistencia completa de todos los cursos: "La hipótesis que se planteó en la observación de ayer tiende a derrumbarse. Los estudiantes de sexto y séptimo asistieron a las clases de hoy y se presentó el mismo fenómeno: los estudiantes no compran productos de la cafetería" (Nota única de análisis en la Observación 9).

De estos hechos, surgen nuevas propuestas hipotéticas; entre ellas: los estudiantes dejan de comprar en la cafetería, ya sea por decisión de ellos o de sus padres, a razón del almuerzo que es ofrecido por el Programa de Alimentación Escolar (PAE). Sin embargo, tal inestabilidad no desdibuja, en ningún momento, el comportamiento de los estudiantes en la fila o en el tránsito por los pasillos de la cafetería: sean pocos los estudiantes o sean muchos los que hagan uso de este espacio son isotópicas las acciones tendientes al juego.

\section{A modo de conclusión}

Este proceso de observación del uso del espacio público en una institución escolar permite comprender las prácticas de los estudiantes dentro de la cafetería. Los propósitos de los estudiantes con el uso de este espacio son variados, pero todos tienden al mismo destino: conciben la cafetería como un espacio para liberar tensiones y emociones, es decir, para jugar, y no solo, como se creería, para comprar productos. Las agresiones de los estudiantes compradores y no compradores resultan gracias a la necesidad del juego y se configuran como expresiones disfemísticas somáticas. Esto indica que no es extraño que los sujetos menores de la institución sean los que más se diviertan al momento del descanso, aunque, para ello, surjan agresiones, insultos y saboteos mutuos cargados de un valor afectivo importante.

Así las cosas, el juego, concepto valorado positivamente por varios investigadores (Pérez y
Collazos, 2007; Alcántaras, Cardoza y Limas, 2013; González, Restrepo y Agudelo, 2014), podría adoptar una concepción negativa en la institución objeto de estudio, si no se comprende la intención disfemística somática que lo caracteriza dentro de las prácticas estudiantiles en la cafetería del colegio.

Por otra parte, las relaciones de poder entre los sujetos que interactúan en este espacio conllevan la comprensión de cierta represión pasional sobre las redes sociales configuradas en la hora del descanso: los que atienden llaman la atención a los estudiantes sobre su comportamiento y deciden también a quién atender y en qué puerta; la organización sugerida tanto por ellos como por los docentes va en detrimento de la configuración del juego que los estudiantes proponen en cafetería.

El hecho de que la concurrencia estudiantil no sea estable da cuenta de la capacidad de compra constante de la que carecen los estudiantes, ya sea por la estratificación social o por el complemento alimenticio del PAE. Independientemente de lo anterior, las filas, sean cortas o extensas, siguen la misma línea de comportamiento estudiantil.

De esta manera, se responde al problema que lleva al proceso de observación: hay constantes llamados de atención para los estudiantes con respecto al mal uso de los espacios reducidos: corren, se golpean, gritan, etc., justamente en la cafetería. Así las cosas, no se trata de que los estudiantes, desde lo que se infiere en los usos que hacen de este espacio, tengan malos comportamientos, sino que utilizan este lugar para jugar y divertirse mientras compran o transitan.

\section{Referencias}

Alcántara, G; Cardoza, S. y Lima, M. (2013). Estudio comparativo del recreo dirigido y el recreo no dirigido y su influencia en la conducta de los y las estudiantes del turno matutino del segundo ciclo de educación básica del centro escolar Jorge Larde del 
municipio de San Martín y el centro escolar San Luis Talpa del municipio de San Luis Talpa (Tesis doctoral). Universidad de El Salvador, San Salvador.

Artavia, J. M. (2014). El papel de supervisión del personal docente durante el desarrollo del recreo escolar. Revista educación, 19-36. Recuperado de: http://www.redalyc.org/arti culo.oa?id=44031370002.

Burbules, N (1989). Una teoría del poder en educación. Revista Propuesta Educativa, año 1, n.1, p. 13-29.

Buritica, D. (2014). El recreo: ¿Es posible pensarlo pedagógicamente? Un camino hacia la prevención de comportamiento agresivo. Tolima: Universidad del Tolima.

Casas Gómez, M. (1986). La interdicción lingüística-Mecanismos del eufemismo y disfemismo. Cádiz: Servicio de publicaciones de la Universidad de Cádiz.

Campos, G., \& Lule, N. (2012). La observación, un método para el estudio de la realidad. Xihmai, 7(13), 45-60. Recuperado de: https://dialnet.unirioja.es/servl et/articulo? codigo $=3979972$.

Carreter, L. (1998): Diccionario de términos filológicos. Madrid: Gredos.

Chaves, A. (2013). Una mirada a los recreos escolares: El sentir y pensar de los niños y niñas. Revista Electrónica Educare, 17 (1), 67-87. Recuperado de:

http://www.redalyc.org/articulo.oa?id=1941 25789005 .

De Castro, M. (2018). El recreo como espaciotiempo de valores. Revista educación en valores, (29), 53-61. Recuperado de: https://dialnet.unirioja.es/servl et/articulo? codigo $=7020956$.
Fontanille, J. (2001). Semiótica del discurso. Lima: Universidad de Lima.

Gold, R. (1958). Roles in sociological field observation. En: Social Forces, 36, 217-213.

González S.; Restrepo C. y Agudelo Ch. (2014). El recreo en la Educación Básica: una pregunta por el juego y la convivencia escolar. (Tesis de Maestría). Universidad Pontificia Bolivariana, Medellín, Colombia.

Greimas, A. y Courtés, J. (1990). Semiótica. Diccionario razonado de la teoría del lenguaje. Madrid: Gredos.

Herrero, M. (1997). La importancia de la observación en el proceso educativo. En: Revista electrónica interuniversitaria de formación del profesorado, (1), 85.

Jarauta, S. (2017). Una mirada al patio: análisis observacional del recreo en educación infantil. Valladolid: Universidad de Valladolid.

López, P. (2017). El recreo o descanso escolar: ¿Escenario educativo para el reconocimiento del otro? Manizales: Universidad Católica de Manizales.

Mandoki, K. (2006). Prosaica uno. México: Siglo XXI Editores.

Peñaranda, C. (2017). El recreo como espacio de interacción: un estudio observacional. Valladolid: Universidad de Valladolid.

Pérez, L. y Collazos, T. (2007). Los patios de recreo como espacios de aprendizaje en las instituciones educativas sedes Pablo Sexto en el municipio de Dos Quebradas (Tesis de pregrado). Universidad Tecnológica de Pereira, Colombia.

Postic, M. y De Ketele, J. (2000). Observar las situaciones educativas. Madrid: Narcea Ediciones. 
Rodríguez, G.; Gil, J. y García, E. (1998). Metodología de la investigación cualitativa. Madrid: Ediciones Aljibe.

Rosenberg, M. J. \& Hovland, C. I. (1960). Cognitive, affective, and behavioral components of attitudes. En: Attitude organization and change (pp. 1-14). New Haven, CT: Yale University Press.

Valbuena, É. (2016). El análisis del contenido: de lo manifiesto a lo oculto. En: $L a$ investigación en Ciencias Sociales. Bogotá: Universidad Piloto de Colombia. 